\title{
Design and Implementation of Watermarking Technique using DWT-DCT for Authentic, Copyright Protected and Secured Cheque Transaction for Bank CTS
}

\author{
Shidramshetti S. Pasarad \\ Student, Department of DECS, \\ VIAT, VTU, CPGS, Bengaluru Region, \\ Muddenahalli, \\ Chikkaballapur, India
}

\author{
Ravi Talawar \\ Assistant Professor \\ Department of DECS, VIAT, VTU, CPGS, \\ Bengaluru Region, Muddenahalli, \\ Chikkaballapur, India
}

\begin{abstract}
Cheques Truncation System can be used For the rapid clearing of cheques. Cheques Truncation System of the bank transmits the electronic version of cheque pictures to drawee branches for the payment through the clearinghouse. We are blindly trusting that the arrangement is very safe and insured. However, genuine information in the cheque image may be damaged and the quality may degrade or may duplicate by the intruders. In this work, Design and Implementation of watermarking technique using DWT-DCT for authentic, copyright protected and secured cheque transaction for CTS of the bank is discussed. In this work, it is implemented imperceptibility of watermark which are supported for copyright protection and security of cheque images.
\end{abstract}

\section{General Terms}

Invisible Watermarking, Secure, Authentication

\section{Keywords}

CTS, DWT, DCT, Copyright protection.

\section{INTRODUCTION}

In the past few years the internet technology has been doing the development of wide network facilities and digital data communication. Data transferred from one device to another device may be in the form of audio, text, video, images etc. The important documents like property papers, military documents, medical documents or any personal documents can be sent one device to another over the internet. Likewise the bank's Cheque Truncation System uses cheque's image as data and transfer it to the drawer branch of the bank for payment through the clearinghouse. In such cases there is a risk of degradation of the image due to the interruption of data being added in the middle of communication media. Consequently the image cannot be a good visible image at receiving bank. Also, it may get copyright by the intruders. Thus, there is a need of authentication, security and copyright protection for cheque images.

There are different ways to provide security to image such as digital watermarking methods, encryption and decryption methods, steganographic method, etc. However the digital watermarking techniques are the best choice for good reproduction, authentication and security purpose. The digital watermarking system can be divided into two main subsystems, they are embedding subsystem and extracting subsystem. Both are complement to each other. In an embedding subsystem the watermark is hidden in the original image to procure watermarked image and measure insensibility of watermarked image (imperceptibility). In an extracting subsystem the output of the embedding subsystem will be the input and the watermark will be extracted and measure robustness of image. The two important properties that are required to be taken care in the digital watermarking are imperceptibility and the robust nature against attacks that try to superannuation or destroying watermark. Many researches in this field are carried out in search of more effective techniques to provide these two properties in watermarking.

\subsection{Methods of Watermarking of Cheque Image:}

Digital watermarking of cheque image is the process in which watermark is embedded in a covered image of bank cheque, to provide authenticity, copyright protection \& cheque security. There are different watermark used for copyright protection such as visible watermark, invisible watermark, private watermark, public watermark, fragile watermark, etc.

Watermarking of cheque can be done by two method, i.e. a) Spatial domain technique and b) transform domain technique [I, 2, 7, and 9]. In spatial domain technique, watermarking of digital cheque image is done on the basis of the pixel location. Since spatial domain technique is very simple and easy wherein covered image is directly replaced by watermark using the substitution method. Spatial domain techniques are very fragile and poorly vigorous to external attack on watermarked image [2, 4, 8, and 11]. In transform domain technique, transform is applied on cover image of the cheque. A watermark is used to embed into middle band frequencies, co-efficient of transforming images of the cheque, and then apply the inverse transforms to get a watermarked cheque image. There are various transforms most widely used like DCT, DFT, SVD, DWT, Gabor transforms, etc. [13, 14, 15, and 16]. In this report, the use of a combination of DWT-DCT for watermarking of cheque images is discussed. Since, the combination of DWT-DCT is vigorous to common attack while doing image processing like noise, filtering \& compression, cropping \& rotation, etc. 


\subsection{Block Diagram}

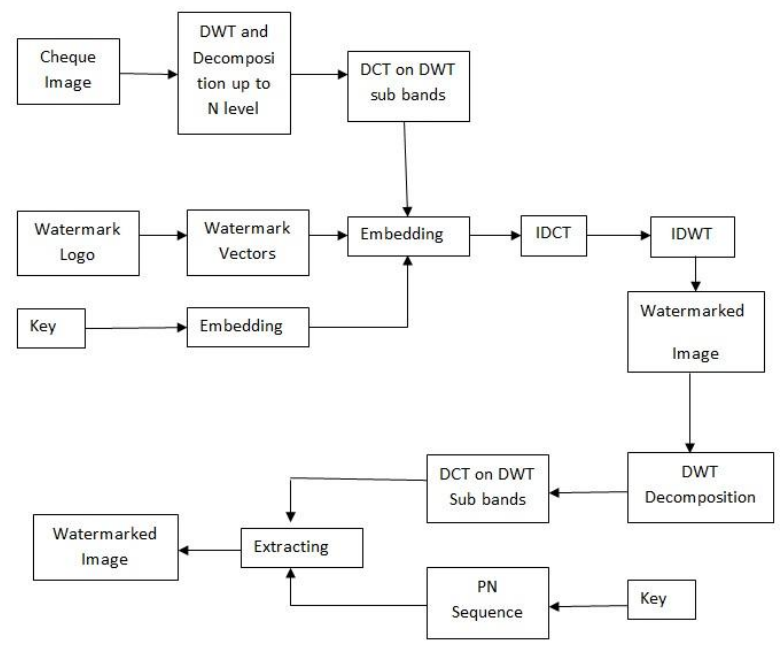

Fig. 1.1 Block diagram of combined DWT-DCT Watermarking technique

Following discussion of wavelets is based on a presentation by the mathematician, whose paper provides a good foundation for understanding wavelets, and includes a number of derivations that are not given here. A wavelet, in the sense of the Discrete Wavelet Transform, is an orthogonal function which can be applied to a finite group of data. Functionally, it is very much like the Discrete Fourier Transform, in that the transforming function is orthogonal, a signal passed twice through the transformation is unchanged, and the input signal is assumed to be a set of discrete-time samples. Both transforms are convolutions.

\section{WORKING OF DWT TRANSFORM}

Discrete wavelets transform consist of discrete sample of wavelets. It has ability to hold both location information and frequency. With the help of this property, the filter is used to divide the bank cheque image in four non-overlapping sub bands like LL, LH, HL, and HH. The LL sub - band is a low pass band and remaining three sub-bands are high pass band: HL denotes horizontal sub-band, LH denotes vertical subband and $\mathrm{HH}$ denotes diagonal sub-band as shown in figure. The coarse scale of DWT co-efficient is represented by LLI sub - bands and the finite scale DWT co-efficient is represented by $\mathrm{LHl}, \mathrm{HLl}$, and HHI sub-bands. The sub-band LLl is processed further and divide into four non-overlapping sub-bands like LL2, LH2, HL2and HH2 to obtain the next coarse scale of DWT co-efficient. This process continues until some final coarse scale of wavelet co-efficient. Energy of the image is concentrated at lower frequency sub-band LLx and therefore embedding watermark in such sub-band may degrade the quality of the image. However, embedding the watermark in these low frequency sub-bands may increase robustness. The edges and texture of the image are included in high frequency sub-band $\mathrm{HHx}$ and human vision is not generally sensitive to change in such sub-band. This property allows the watermark embedding without being perceived by the human vision. Many DWT algorithms used for digital watermarking is to implant the watermark in the middle frequency sub-bands LHx and HLx from where robustness, imperceptibility performance can be achieved.

$\mathrm{L}$ and $\mathrm{H}$ denotes Low and High pass filters respectively, 2 indicates subsampling. The output of this filter is given as equations 2.1 and 2.2 [19].
$\mathrm{Ai}+1[\mathrm{p}]=\sum_{n=-\infty}^{+\infty} L(n-2 p) A i[n]$

$\operatorname{Di}+1[\mathrm{p}]=\sum_{n=-\infty}^{+\infty} H(n-2 p) A i[n]$

Elements $\mathrm{Ij}$ are using for the next steps (scaling) of the transformation and $\mathrm{Dj}$ elements are called as wavelet coefficients which determines the output of the wavelet transform. $\mathrm{L}[\mathrm{n}]$ and $\mathrm{H}[\mathrm{n}]$ are coefficients of low and high pass filters respectively. One can assume that on scaling $\mathrm{j}+1$, there is only half from the number of $\mathrm{A}$ and $\mathrm{D}$ elements on scaling $\mathrm{j}$. This causes that DWT can be done by only $2 \mathrm{Aj}$ elements remained in analyzed signal. These elements are called scale function coefficients.

DWT algorithm for 2-dimensional pictures is shown. First DWT is performing for an image rows and then for columns (Fig. 2.1) [19].

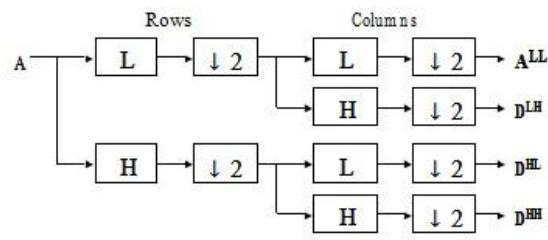

Fig. 2.1 Wavelet decomposition for 2-dimensional picture

\begin{tabular}{|c|c|}
\hline $\begin{array}{c}\text { LL } \\
\text { Approximate } \\
\text { Sub-band }\end{array}$ & $\begin{array}{c}\text { HL } \\
\text { Horizontal } \\
\text { Sub-band }\end{array}$ \\
\hline $\begin{array}{c}\text { LH } \\
\text { Vertical } \\
\text { Sub-band }\end{array}$ & $\begin{array}{c}\text { HH } \\
\text { Diagonal } \\
\text { Sub-band }\end{array}$ \\
\hline
\end{tabular}

Fig. 2.2 DWT Sub-bands in general Watermarking Method.

Haar DWT is calculated by cascading of filters followed by factor 2 sub-band subsampling (Fig. 2.3). Where in each level of DWT, the Approximate sub-band is going to sampled as shown [19].

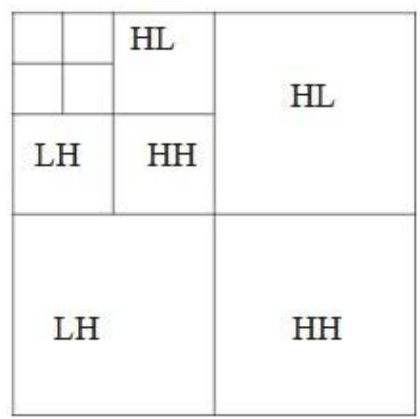

Fig. 2.3 2-level Sub-band image after sampling

Implementing The Wavelet Transformer The design of the Wavelet Transform was carried out in six stages, as follows:

1. Simulate the wavelet lattice filters in Matlab to understand the basic operation of the wavelet transformation, and write $\mathrm{C}$ 
code to determine the proper implementation for a multidilation wavelet transformer. Ideally, the basic block-diagram structure of the transformer should remain the same for varying orders of wavelet functions, varying wavelet coefficients, varying input block sizes, and should also maintain the same structure between the decomposition and recomposition filters.

2. Design a simple lattice filter and confirm its function.

3. Create modules or subroutines to perform the operations of the complete wavelet transform.

4. Create the complete transformer and confirm its function. A possible simple implementation is to have one filter to decompose an input into its wavelet representation, with its output feeding directly into another filter in order to recompose the signal back into its original form. The output can be checked against the input for various signals such as sine waves, square waves, and speech.

5. Optimize the wavelet transformer routines such that the total computation time per input sample-time is as small as possible.

6. Write software to automatically design lattice filters, given certain parameters such as wavelet coefficients, input block size, and number of dilations to decompose the input.

\section{DISCRETE COSINE TRANSFORM}

Discrete Cosine Transform (DCT), Relationship in-between DCT and FFT, Discrete Cosine Transform is actually a cutdown variant of the Fourier Transform or the Fast Fourier Transform:

- DCT considers the real part of FFT only

- DCT is computationally simpler than FFT

- DCT: Effective for multimedia Compression

- DCT is most normally used (than FFT) in multimedia Image/Video Compression.

- Cheap MPEG Audio Variant: more later.

\subsection{Applying the DCT:}

- Similar to the discrete Fourier transform: - DCT transforms a image and/or signal from the time domain to the frequency domain - DCT can approximate lines well with fewer coefficients,

- Helps separate the image into spectral sub-bands of differing importance (with regard to the image's visual quality).

\subsection{Working of DCT Transform:}

A discrete cosine transform (DCT) can also be expressed as a sum of cosine functions oscillating at different frequencies which has a sequence of finitely many data points $[1,4,6,8]$. The equation of discrete cosine transforms and definition of DCT Regions is as shown below:

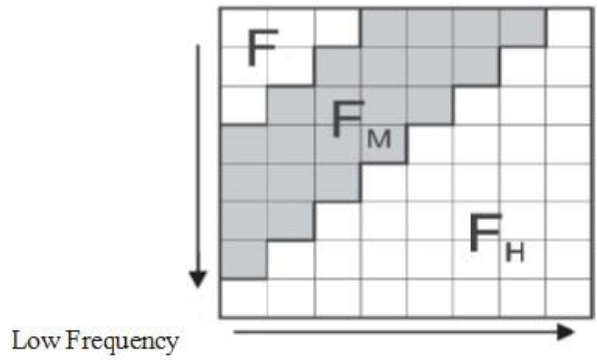

High frequency

Fig.3.2.1 Definition of DCT Regions used to embed the watermark.

2D-DCT:

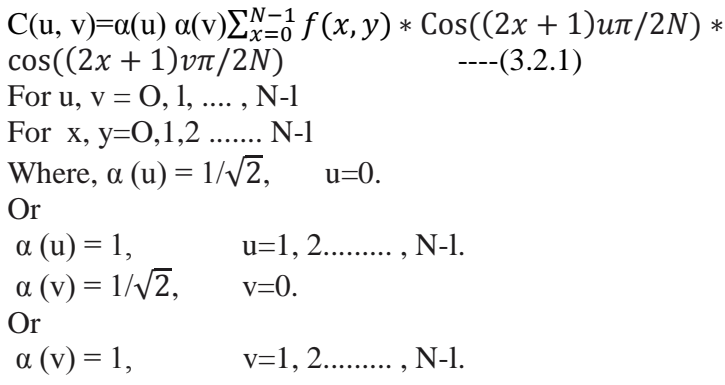

2D-1DCT:

$f(x, y)=\sum_{u=0}^{N-1} \alpha(\mathrm{u}) \alpha(\mathrm{v}) \mathrm{C}(\mathrm{u}, \mathrm{v}) * \operatorname{Cos}((2 x+1) u \pi / 2 N) *$ $\operatorname{Cos}((2 x+1) v \pi / 2 N) \quad-----(3.2 .2)$

FL is used to denote the lowest frequency components of $n$ the block, while $\mathrm{FH}$ is used to denote the higher frequency components. FM is chosen as the embedding region as to provide additional resistance to lossy Compression techniques, while avoiding significant modification of the cover image $[17,18]$.

\subsection{Performing DCT Computations:}

The basic functionality of the DCT is as follows:

- The input image is divided into $\mathrm{N}$ by $\mathrm{M}$ matrix form;

- $f(i, j)$ is the intensity of the pixel in a row $i$ and column $j$;

- F $(u, v)$ is the DCT coefficient in row ui and column vj of the DCT matrix.

- For JPEG image (and MPEG video), the DCT input is usually an 8 by 8 (or 16 by 16) array of integers. The array contains each image window's respective colour band pixel level; 


\section{METHODS OF WATERMARKING OF CHEQUE IMAGE \\ 4.1 Data Flow Diagram Of Watermarking}

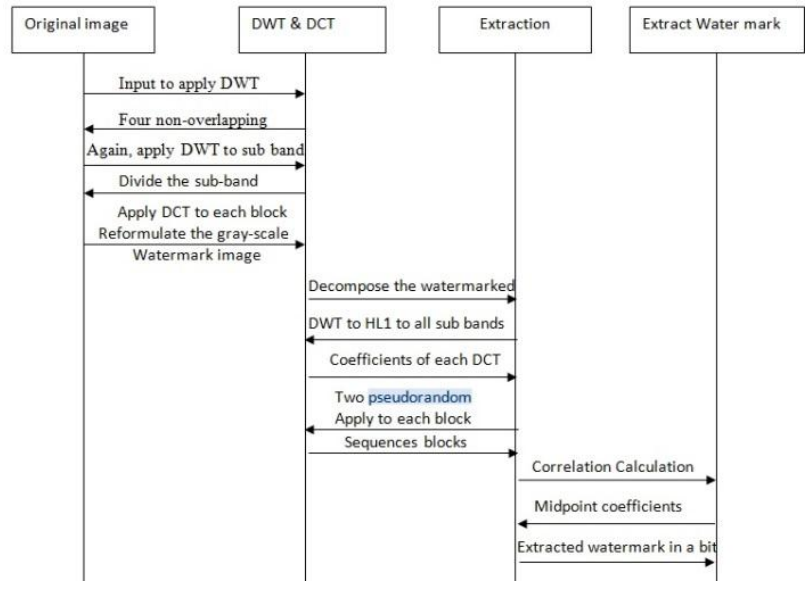

Fig 4.1 Data flow diagram of DWT-DCT watermarking technique for CTS

Digital water marking of cheque image is the process in which watermark is embedded in a covered image of bank cheque, to provide authenticity, copyright protection \&cheque security. Watermarking of cheque can be done by two methods. a) Spatial domain technique and b) transoms domain technique $[1,2,7,9]$,. There different watermark used for copyright protection such as visible watermark, invisible watermark, private watermark, public watermark, fragile watermark, etc.

In this work, it IS going to discuss the invisible digital watermarking scheme used copyright protection and security of bank cheque.

\subsection{Watermark Embedding Process:}

Step 1: Select the bank cheque image $[2,9]$.

Step 2: Decomposed the cover image of bank cheque into non-overlapping multi resolution sub-division bands like LLl, $\mathrm{LHl}$, and $\mathrm{HLl}$ and $\mathrm{HHl}$ on applying DWT $[2,9]$.

Step 3: Again decomposed the non-overlapping multi resolution sub-division bands i.e. HLl sub-division bands are divided into four smaller sub-division bands and HL2band are selected $[2,9]$

Step 4: The sub-division band HL2 is divided into $4 \times 4$ blocks $[2,9]$.

Step 5: Discrete cosine transform is applied to every block in selected sub-division band HL2 [2, 9].

Step 6: gray scale image with watermark is re-formulated into vector of one's \& zeros [2,9].

Step 7: Create two pseudorandom sequences which are not similar to each other. One sequence is used to insert watermark bit 0 and other sequence is used to insert watermark bit 1 . The elements present in PN sequence should be exactly equal to the number of middle band frequency elements of DCT transformed DWT sub-bands [2,9].

Step 8: PN_O and PN_I are the two pseudorandom sequences are used for embedding processing with the help of gain factor $\mathrm{k}$, in the DCT transformed 4x 4 blocks of selected DWT HL2 sub-bands of the cover image of bank cheque $[2,9]$.

A watermark is not embedded in all frequencies of DCT coefficient. If we consider matrix $\mathrm{Z}$ containing middle band frequency coefficients of the discrete cosine transformed block, then it carries out the embedding process as shown in equation below.

If the watermark bit is 1 then

$\mathrm{Z}^{\prime}=\mathrm{Z}+\mathrm{k} * \mathrm{PN} \_\mathrm{I}$

Otherwise,

If the watermark bit is 0 then

$\mathrm{Z}^{\prime}=\mathrm{Z}+\mathrm{k} *$ PN_O

Step 9: After modification of its middle band frequency coefficient, inverse discrete cosine transform is applied for embedding watermark bits [2, 9].

Step 10: To produce the watermark cover image of bank cheque the inverse discrete wavelet transform is applied on the DWT transformed image of the cheque which includes the modified sub-division bands $[2,9]$.

\subsection{Flow Diagram For Watermark Embedding Process:}

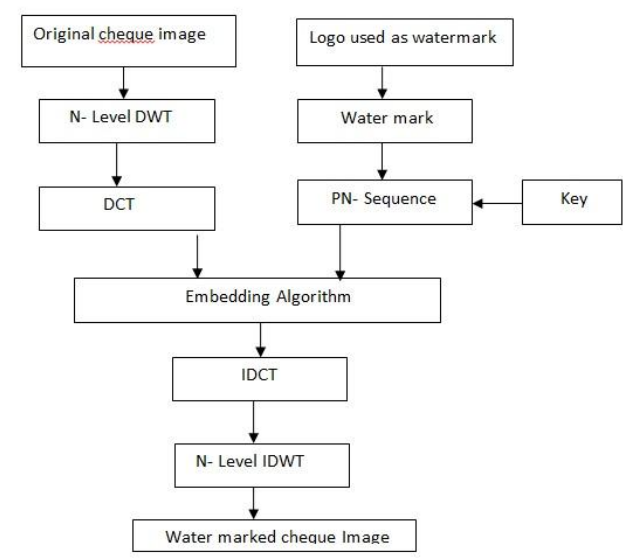

Fig. 4.2 Watermark embedding process

The algorithm and diagram represent the extraction procedure of watermark using combined DWT -DCT technique. In this algorithm, combined DWT-DCT watermark extraction technique used for the cheque image is explained using2-level DWT is used while extracting the watermark logo. In diagram n-level DWT is explained because software having facilities to decomposing the cheque image up to n-level where $n=1,2$, $3,4,5,6$ and 7 which are the level of DWT decomposition of cheque image.

\subsection{Watermark Extraction Process:}

Step 1: Select bank's watermarked cheque image $[2,9]$.

Step 2: Decomposed the watermarked cheque image of bank into four different bands which are non-overlapping multi resolution band such as LLI, LH2, HLl, and HHI on applying DWT $[2,9]$.

Step 3: Again decomposed the non-overlapping multi resolution sub-bands i.e. HLl sub-division band is divided into four smaller sub-division bands and HL2 band are selected [2, 9]. 
Step 4: HL2 sub-division band is divided into 4x 4 blocks [2, 9].

Step 5: Middle band frequency coefficients from each block of the DCT transformed image are extracted from selected sub-division band of HL2 after DCT is applied to each block $[2,9]$.

Step 6: The same seed is used to create again PN 0 and PN_1 as two pseudorandom sequences which were utilized in the watermark embedding process $[2,9]$.

Step 7: Create two pseudorandom sequences PN_O and PN_1 and the correlation between the middle band frequency coefficient is calculated for each block in the sub-division band of HL2.The extracted a bit of watermark is consider as 1 if the correlation with PN_O is lower than PN_1, if not extracted bit of watermark is considered as $0[2,9]$.

Step 8: Calculate the similarity between the original watermark and extracted watermark by reconstruction of the watermark using extracted watermark bits $[2,9]$.

\subsection{Flow Diagram For Watermark Extraction Process:}

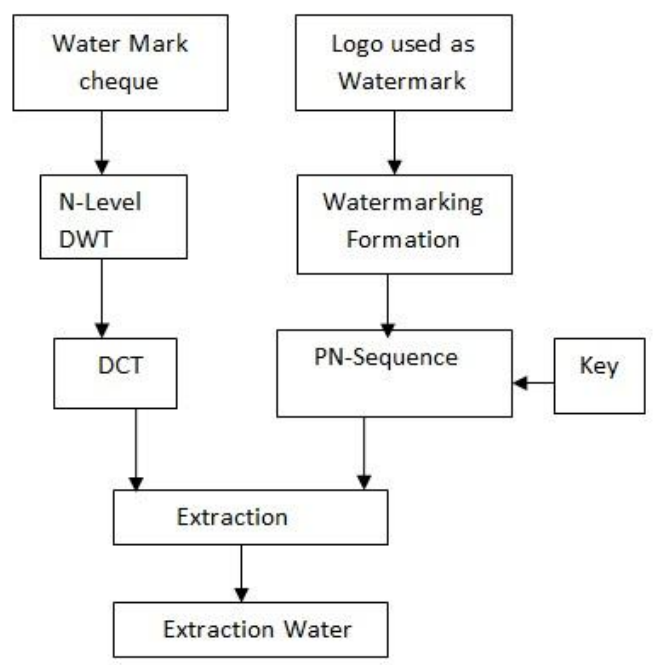

Fig. 4.3 Watermark extraction process

\subsection{Working Of Dwt-Det Watermarking Used For Bank Cts:}

DWT-DCT watermarking software used for bank CTS requires Window XP, language like Matlab R2010a, Dot Net3.0 framework, compiler of Matlab R2010a \& hardware as ram of $1 \mathrm{~GB}$ or higher. To run the software, it requires two inputs, i.e. Cover image of bank cheque and the watermark that is used to embed in that bank cheque image as shown below.

The following are steps for execution of Digital watermarking embedding \& extraction software used for CTS of bank cheque $[1,6,10,12,13]$.

Step 1: Run the software.

Step 2: Select the cover image of bank cheque by clicking on a browse cover image button.

Step 3: Browse or select watermark as message image or logo image for embedding purpose.

Step 4: Select the levels of discrete wavelet transform according to requirement and data type like an unsigned integer for black and white image of bank cheque while data type like double is used for color image of bank cheque.

Step 5: Perform discrete wavelet transform for image.
Step 6: Create sub-band of cheque image for embedding watermark after performing discrete wavelet transform.

Step 7: Finally, DCT is applied at the sub-bands of DWT for the successful watermark embedding process.

Step 8: Save the watermarked cheque image.

Step 9: After the complete watermark embedding process watermarked cheque is transferred to a drawer branch of the bank through the clearing house. Thus, watermark provides the security and copyright protection to bank check.

Step 10: After receiving the watermarked cheque to drawer branch, it will select a watermarked image of cheque with the help of extracting part of digital watermarking embedding \&extraction software.

Step 11: After selection of watermarked cheque image, click on the watermark extraction button to extract the watermark from the cheque.

Step 12: After extraction of watermark logo from a watermarked cheque image, we get extracted watermark logo and the original cheque image from which we can calculate the imperceptibility and robustness of the watermark by comparing the extracted watermark logo with original watermark logo.

\section{SIMULATION AND RESULTS}

\subsection{Input And Output Images}

To run the software, it requires two inputs, i.e. Cover image of bank cheque (Fig.5.1) and watermark (Fig.5.2) that is used to embed in that bank cheque image as shown below.

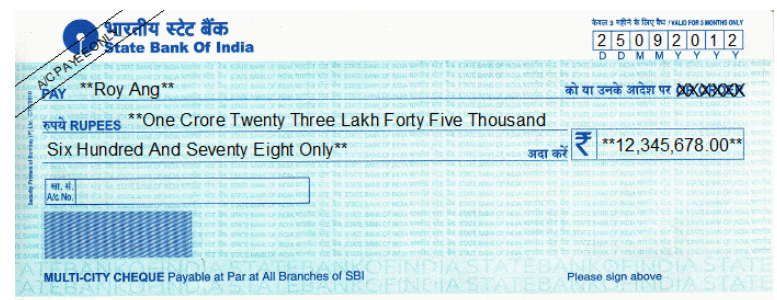

Fig.5.1.Cover Image of Bank Cheque.

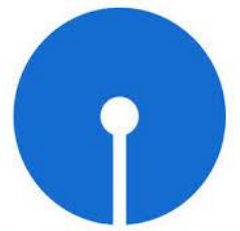

State Bank of India

Fig.5.2 Watermark Image used for Embedding.

\subsection{Calculating Imperceptibility}

To calculate the imperceptibility, by using the following equation. As a criterion of the quality of a watermarked image, PSNR (Peak Signal to Noise Ratio) is used. To calculate the Peak signal to noise ratio, firstly we need to calculate the MSE (Mean Square Error) as follows

$\operatorname{MSE}=\left(1 /\left(\mathrm{m}^{*} \mathrm{n}\right)\right) \sum_{i=0}^{m-1} \sum_{j=0}^{n-1}\|I(i, j)-K(i, j)\|^{2}---(5.1)$

Where $\mathrm{I}$ is the original image, $\mathrm{K}$ is the watermarked image that contains $m$ by $n$ pixels, 
Peak Signal Noise Ratio is calculated according to (5.2):

$$
\begin{aligned}
\text { PSNR dB } & =10 . \log _{10}\left\{\mathrm{MAX}^{2} / \mathrm{MSE}\right\} \\
& =20 . \log _{10}\left\{\mathrm{MAX}^{2} / \sqrt{\mathrm{MSE}}\right\}
\end{aligned}
$$

Here, MAX is the maximum pixel value of the image. Once the PSNR exceeds some value, the errors become undetectable to human viewers. Conversely, the human visual system seems to have a saturation effect as well. Once the image quality falls below a certain level, the image simply looks bad. Watermarked image quality is measured in terms of imperceptibility. The cover image of cheque quality should not disturb due the presence of a watermark. However, peak signal to the noise ratio i.e imperceptibility is calculated in decibels $(\mathrm{dB})$.

\subsection{Observation Table}

\begin{tabular}{|l|c|c|c|c|}
\hline $\begin{array}{c}\text { S1. } \\
\text { No. }\end{array}$ & Image & $\begin{array}{c}\text { DWT levels } \\
1 \text { to } 2\end{array}$ & $\begin{array}{c}\text { PSNR } \\
\text { (Combined } \\
\text { DWT DCT) }\end{array}$ & Gain Factor \\
\hline 1. & SBI Cheque & At all Levels & 39.1883 & $2,4,8$ \\
\hline 2. & $\begin{array}{c}\text { Syndicate } \\
\text { Bank of Wiki } \\
\text { Cheque }\end{array}$ & At all Levels & 47.9506 & $2,4,8$ \\
\hline 3. & $\begin{array}{c}\text { HDFC Bank } \\
\text { Cheque }\end{array}$ & At all Levels & 51.8081 & $2,4,8$ \\
\hline 4. & $\begin{array}{c}\text { Canara Bank } \\
\text { Cheque }\end{array}$ & At all Levels & 53.3876 & $2,4,8$ \\
\hline
\end{tabular}

Table 5.1Table shows Gain Factors and PSNR for Some Bank Cheques

The observation table 5.1 shows the PSNR value for all levels of DWT for different gain factor for same watermark used for different bank cheque images. Profit factor is the condition utilized for powering embedding of watermark. As the value of the gain factor increases the embedding power of watermark in the top image of cheque increases. It is found that PS NR value is same for all levels of DWT \& gain factor.

Following diagram shows the software snapshots screen which is used for Digital watermarking embedding and extraction procedure.

Figure 5.3 explains selection of DWT levels and data type used for cheque image for embedding the watermark for copyright protection using this software.

In this process Browse cover image button is utilized to take the cheque image. Browse MSG image button is utilized to pick out the watermark logo of bank which is used for embedding process.

As image has two data types depending on its feature. Such as color image has data type as double and for black and white or grayscale image has unsigned integer.

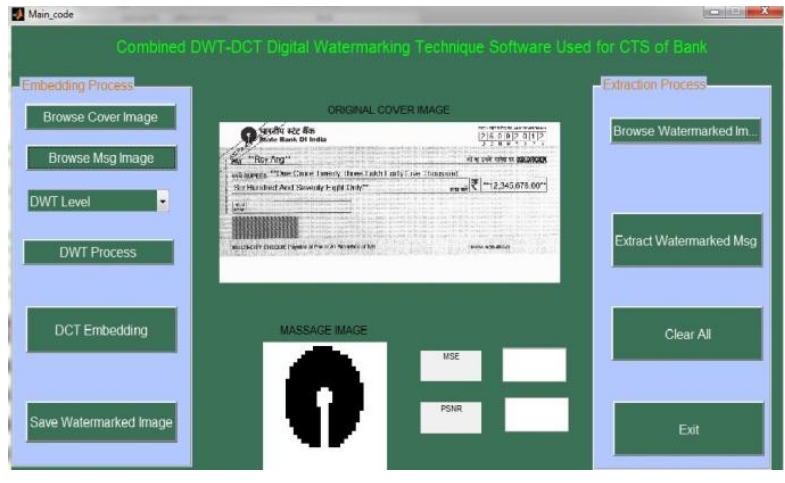

Fig. 5.3 GUI showing cheque and logo of a bank

Figure 5.4 explains working of discrete wavelet transform after applying on cheque image for embedding the watermark logo. In this figure, 2-level of discrete wavelet transform is presented.

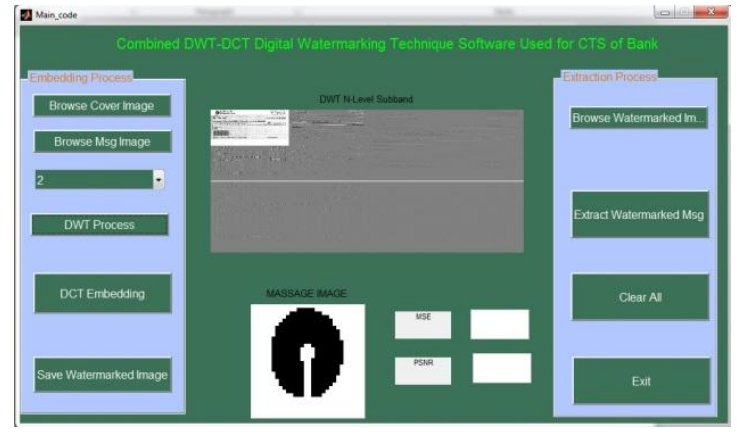

Fig.5.4 Apply DWT on cheque image for embedding the watermark.

Figure 5.5 explains the successful watermark embedding process with the help of sub band of DWT and DCT embedding. In this process, Sub band D WT button is used for to create the sub band of cheque image after using the discrete wavelet transform.

On clicking DCT-Embedding button successful watermark logo is embedded which is indicated in the dialog box after the successful embedding process.

Figure 5.6 explains the extraction process of watermark logo from watermark cheque. The extraction process is generally occurring on receiving side, i.e. At clearing house.

In this process watermarked cheque image is chosen by ticking on the Select WM Img button. Subsequently clicking on Watermark Extraction button watermark logo get extracted. After extraction of watermark peak signal to the noise ratio, i.e. Imperceptibility is calculated for different bank cheques as shown in observation table. Exit button is used for closing the software which is shown in figure-5.6. 


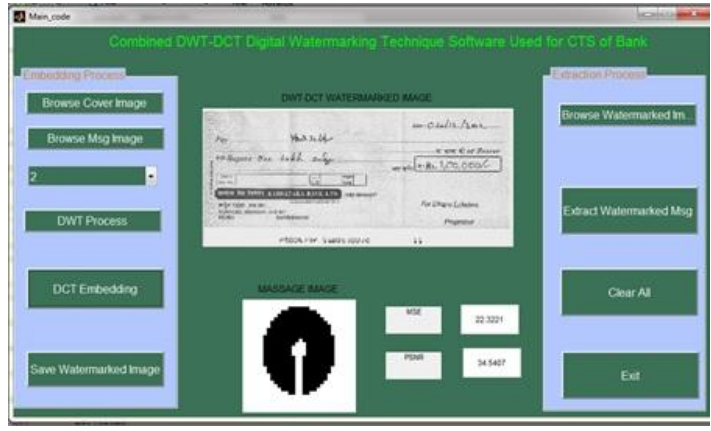

Fig. 5.5 Successful watermark embedding process with the help of sub band of DWT and DCT -embedding.

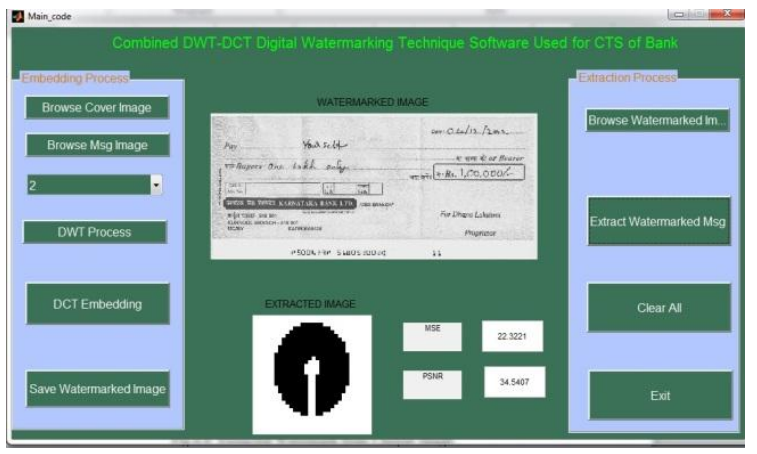

Fig.5.6. Extracted Watermark from Cheque Image.

\section{CONCLUSION}

Thus, working of DCT-DWT Digital Watermarking Technique Software Used in Cheque Truncation System of bank is explained in this report. The DCT and DWT provide high robustness and imperceptibility to the cheque image. This software offers security, copyright protection and data authenticity to cheque image. In this report, it also describes algorithm of digital image watermarking based on combining two transforms, viz DWT and DCT. Watermarking is done by altering the wavelet coefficients of carefully chosen DWT sub-bands, followed by the application of the DCT transform on the chosen approximate sub-bands.

\section{FUTURE WORK}

Although most work in the study of digital image watermarking focuses on utilizing DWT due to its excellent spatial-frequency location and multi resolution properties, which are similar to the theoretical models of the human visual system. Nevertheless, there are two drawbacks associated with DWT. Foremost, it lacks shift invariance, which signifies a little switch in input signal that can have large changes in the energy distribution of the wavelet coefficients. Second, the DWT has poor directional selectivity, which is apparent from the impulse responses of the filters of the individual sub bands. Provides flexible multi resolution representation of pictures. One of the singular attributes of the DCT is that we can determine the number of directional decompositions required at every layer of the multi - resolution pyramid. The ground for applying the two transforms is based along the fact that the combination of transforms could compensate for the drawbacks of each other, resulting in effective watermarking.
DCT began to gather some interest in capability of capturing directional information such as smooth contours and directional edges. In my study since this report has provided PSNR of about 40 to $60 \mathrm{~dB}$ that produces even less visibility after extraction of the icons. The same process of DWT-DCT is the best approach to get increased PSNR to make still robust, improved imperceptibility.

\section{REFERENCES}

[I] MahaswetaJJoshil, Prof. Zankhana H.Shah2. "Watermarking in DCTDWTDomain." Et AI, / (IJCSIT) International Journal of Computer Science and Information Technologies, Vol 2 (2), 2011,717-720.

[2] AM. Kothari I, AC.Suthar2, R.S.Gajre3. "Performance Analysis of Digital Image Watermarking TechniqueCombined DWT -DCT over individual DWT.", Published in International Journal of Advanced Engineering \& Applications, Jan. 2010.

[3] S SBedi, Ashwani Kumar, and PiyushKapoor. "Robust Secure SVDBased DCT - DWT Oriented Watermarking Technique for Image Authentication." Special Issue of the International Journal of the Computer, the Internet and Management, Vol.17 No. SPI, March 2009.

[4] Ben Wangl, Jinkou Ding2, QiaoyanWenl, XinLiaol, CuixiangLiul."An Image Watermarking Algorithm Based on DWT DCT and SVD.",Proceedings of ICNIDC2009.

[5] Saeed K Amirgholipour, Ahmad R. Naghsh-Nikhi. "Robust Digital Image Watermarking Based on Joint DWT-DCT."Published in International Journal of Digital Content, Technology and its Applications, Volume 3, Number 2, June 2009.

[6] Ruiling Zhu, Xin Wang. "Efficient Digital Watermarking in DCTDomain." School of Information Science and Engineering, ShandongUniversity, Jinan, Shandong, 250062, China 2009 International Forum on Information Technology and Applications.

[7] Me Jianshengl, Li Sukangl and Tan Xiaomei2. "A Digital Watermarking Algorithm Based On DCT and DWT." Nanchang Power Supply Company, Nanchang, China Proceedings of the 2009International Symposium on Web Information Systems and Applications (WISA'09) Nanchang, P. R. China, May 22-24, 2009, pp. 104-107.

[8] Liwei Chen, Mingfu Li. "An Effective Blind Watermark Algorithm Based on DCT." Proceedings of the 7th World Congress on Intelligent Control and Automation June 25 - 27, 2008, Chongqing, China.

[9] Ali AI-Haj. "Combined DWT-DCT Digital Image Watermarking."Department of Computer Engineering, School of Electrical Engineering, Princess Sumaya University for Technology, PO Box 1928, AI-Jubeiha,

11941 Amman, Jordan Journal of Computer Science 3 (9): 740-746,2007 ISSN 1549-3636 C) 2007 Science Publications.

[10] Reddy, A and B. Chatterji, 2005. "A New Wavelet Based Logo watermarking Scheme," Pattern Recognition Letters, 26 (7): 1019-1027. 
[11] Tsai, M. And H. Hung, 2005. "DCT and DWT based Image Watermarking Using Sub sampling," in Proc. Of the 2005 IEEE Fourteenth. Conf. On Machine Learning and Cybernetics, pp: 5308-5313, China.

[12] Wang, S. and Y. Lin, 2004. "Wavelet Tree Quantization for Copyright Protection Watermarking," IEEE Trans. Image Processing, Vol 13, no. 2, pp: 154-164.

[13] Chan, C. And L. Cheng, 2004. Hiding Data in Images by Simple LSBSubstitution, Pattern Recognition, 37 (3): $469-474$.

[14] Nikolaidis, A. And I. Pitas, 2003. "Asymptotically optimal detection for additive watermarking in the DCT and DWT domains," IEEE Trans. Image Processing, 2 (I 0): $563-571$.

[15] Lin, S. And C. Chin, 2000. "A Robust DCT -based Watermarking for Copyright Protection," IEEE Trans. Consumer Electronics, 46 (3): 415 -421.
[16] Juan R. Hernandez, Fernando Perez-Gonzalez, Martin Amado. "DCTDomainWatermarking Techniques for Still Images: Detector Performance Analysis and a New Structure." IEEE Transaction on Image Processing, vol. 9, no.I, January 2000 .

[17] Nidal F. Shilbayeh, Belal AbuHaija, and Zainab N. AlQudsy. "Combined DWT-CT Blind Digital Image Watermarking Algorithm". International Science index. Vol.7, No: 6,2013.

[18] Baisa L. Gunjal and Suresh N.Mali. " Secured color image watermarking Technique in dwt-dct domain". International Journal of Computer Science, Engineering and Information Technology (IJCSEIT), Vol.1, No.3, August 2011.

[19] Marcin Kociołek, Andrzej Materka, Michał Strzelecki, Piotr Szczypiński,have puplished "Discrete Wavelet Transform Derived Features For Digital Image Texture Analysis". 